\title{
FOCUS ON TRANSLATIONAL RESEARCH
}

\section{MPMI Focus Issue Overview}

Readers of this journal are well aware how far our field has progressed towards mechanistic understanding of pathogenesis and immunity. Almost concurrently, this new fundamental knowledge is being translated into new tools for reducing disease losses in crops. This is obviously exciting for our research community and our varied stakeholders. Thus, the MPMI editorial board decided that the time is right for a special issue devoted to the topic of Translational Research. This issue comprises reviews, invited by the editors, and primary reports submitted in response to an open call. We are gratified to introduce articles that collectively encompass a wide range of applications and clearly illustrate the practical value of fundamental understanding of molecular plant-microbe interactions.

Plant immune surveillance is among the most intensively studied aspects of plant-pathogen interactions, dating back to Flor's pioneering genetic studies and continuing with molecular, biochemical, and structural studies that are bringing mechanisms into focus. We now know that plants can recognize pathogen-associated molecular patterns and secreted effector proteins as signals of invasion. This fundamental understanding provides a foundation for efforts to develop more efficient, durable genetic resistance in crops. Lloyd and colleagues describe how assays for PAMP-triggered immunity (PTI), first developed in model systems, can be adapted for use in a crop (canola, Brassica napus). They use these assays to demonstrate substantial variation in PTI among different B. napus cultivars, which can be exploited in breeding programs to enhance PTI. Zuluaga Cruz and coworkers describe a different tool for breeding resistance to a bacterial wilt pathogen of potato: the bacterium is transformed with a luciferase transgene, enabling quick, quantitative screens for resistance based on light emission. This simple approach will facilitate screens for resistance against this vexing pathogen.

Stirnweis and colleagues describe an effort aimed at enhancing the efficiency of effector-triggered immunity. They use targeted mutagenesis, informed by natural variation in resistance $(R)$ gene alleles, to expand the recognition specificity of a wheat $R$ gene against powdery mildew. Their approach provides a general blueprint through which nucleotide binding-leucinerich repeat genes against diverse pathogens could be enhanced. Sorel and colleagues review the current state of knowledge of the cylindrical inclusion $(\mathrm{CI})$ protein encoded by potyviruses. The authors emphasize recently discovered roles in ETI as well as in subversion of resistance conditioned by recessive resistance genes. Thus, studies of this relatively neglected viral protein may lead to greater understanding of host-plant resistance and aid in the development of new approaches to resistance.

To cope with the plant immune system, successful pathogens have evolved to avoid or sabotage host surveillance. We are now beginning to develop a mechanistic view of how this occurs, and genome sequences are providing a powerful tool through which knowledge from model systems can be applied to less tractable crop pathosystems. This is illustrated in a comprehensive review of cereal rusts by Hulbert and Pumphrey, who describe new approaches to combat these dire threats to food security and also offer examples of unique biology that can be uncovered in these systems. Yin and colleagues illustrate how a sequenced genome can be exploited to reveal a new virulence mechanism in a challenging experimental system. This paper also illustrates the utility of host-induced gene silencing, through which dsRNA from a plant transgene activates RNAi against a specific gene in the pathogen. This approach holds great promise for basic research on nontransformable pathogens and for disease control in the field. Another example of the utility of comparative genomics is contributed by Lee et al., who leverage previous studies from rice and tomato pathosystems to establish how the PAMP chitin is perceived by wheat and how this perception is subverted by a chitin-binding effector secreted from a fungal wheat blotch pathogen.

Effector biology has been one of the most active areas of molecular plant-microbe research in recent years. Much has been learned about pathogen virulence and host resistance from these efforts. This knowledge has inspired a new approach for resistance breeding based on the gene-for-gene paradigm, in which effectors identified from pathogen genome sequences are used as probes for novel $R$ genes. This approach typically involves delivery of effectors from a surrogate pathogen, thereby increasing throughput and expanding the range of potential resistance sources beyond what was previously possible with pathogen-based screens. The utility of effectors as tools for resistance breeding is comprehensively reviewed by Vleeshouwers and Oliver. Meanwhile, Upadhyaya et al. describe a specific application of this approach to the aforementioned wheat rust problem.

Fundamental understanding of pathogen biology is also fueling novel strategies that extend beyond improving host immunity. Lindow and colleagues describe one such approach in which bacteria causing Pierce's disease are 'confused' by transgenic overexpression in the plant of a gene for a diffusible signal factor that regulated bacterial attachment. Another innovative technique was introduced by Montero-Astúa and coworkers, who show how expression of a viral glycoprotein in transgenic tomato could effectively trap the virus in the plant host, thereby inhibiting transmission by the insect vector. Presumably, similar approaches could be applied to protect plants against other insect-transmitted viruses.

MPMI focus issues are designed to bring attention to important research areas, influence research directions, and provide resources for teaching and outreach. We hope to have achieved those goals, and we sincerely thank the authors for contributions that illustrate the enormous potential of translational research in molecular plant-microbe interactions.

Guest Editors

John M. McDowell, John Carr, and Matteo Lorito 